\title{
New blood for old? High quality evidence that fresh red blood cells confer no benefit for critically ill patients
}

\author{
Timothy S. Walsh ${ }^{1}$ and Nicole P. Juffermans ${ }^{2^{*}}$ (1)
}

(c) 2018 Springer-Verlag GmbH Germany, part of Springer Nature and ESICM

Anaemia is prevalent among critically ill patients, and $20-40 \%$ receive red blood cell (RBC) transfusions [1]. Evidence shows us that for most patients, restrictive (RBC) transfusion represents best practice [2]. One reason for this is that we increasingly recognise that RBCs might have harmful as well as beneficial effects. Understanding the risk-benefit balance between tolerating anaemia and treating it with RBCs remains a key question in healthcare. Changes that occur during RBC storage are a potential key modifier of this risk-benefit balance.

After donation, RBCs can be stored for 35-42 days. To minimise wastage, blood banks typically issue the oldest units first and the average transfused storage age is 18-21 days in most blood services. The permitted storage duration is mainly defined by demonstrating $75 \%$ recovery of transfused RBCs in the recipient circulation $24 \mathrm{~h}$ post-transfusion [3], but this measures survival of RBCs and not whether they can carry oxygen to tissues (which is why clinicians give them). During storage, a plethora of structural and biochemical changes occur which, from a pathophysiological perspective, decrease oxygen carrying capacity and might be harmful (Fig. 1). Stored RBCs may modulate host immune response and impair microcirculatory flow $[4,5]$, both clearly detrimental during critical illness. Numerous observational cohort studies have explored the association between RBC storage and a range of outcomes, both in critical illness and other populations [6]. The findings are heterogeneous, with many suggesting harm from older RBCs, but this research is

\footnotetext{
*Correspondence: n.p.juffermans@amc.uva.nl

${ }^{2}$ Department of Intensive Care and Laboratory of Experimental Intensive Care and Anesthesiology, Academic Medical Center, Room G3-206,

Meibergdreef 9, 1105 AZ Amsterdam, The Netherlands

Full author information is available at the end of the article
}

subject to major confounding and in isolation should not change practice. The organisational and financial implications of supplying fresher RBCs are enormous and require high quality evidence. Storage time is not the only potential influence on the risk-benefit balance from RBC transfusion. Donor characteristics such as age and gender have also been associated with recipient outcomes [7], and the manufacturing processes itself (such as the storage medium) may influence $\mathrm{RBC}$ properties when transfused [8].

It is remarkable that until 2015 we had no randomised controlled trials (RCTs) including more than 100 patients addressing the question of storage lesion, but this has changed during the past 3 years.

In this issue of Intensive Care Medicine, Rygard et al. present a systematic review and meta-analysis of trials comparing shorter versus longer $\mathrm{RBC}$ storage time in adult critical care [9], including the TRANSFUSE trial, published very recently. This high quality review conforms closely to Cochrane and PRISMA guidance. The primary outcomes of interest were mortality, adverse events and post-transfusion infections; observational studies have suggested that all of these may be increased by transfusing older RBCs. In additional to estimating overall effect sizes, the authors used a technique called trial sequential analysis (TSA), which quantifies the statistical reliability of data in a cumulative meta-analysis. This technique protects against spurious type I or II error, and allows an estimation of how conclusive evidence is for pre-specified effect sizes. The authors included seven trials in 18,283 patients. Almost all patients were in three large RCTs: ABLE $(N=2510)$, INFORM $(N=10,578)$, and TRANSFUSE $(N=4994)$. Importantly, all trials used leucodepleted RBCs and in the two trials

\section{Springer}




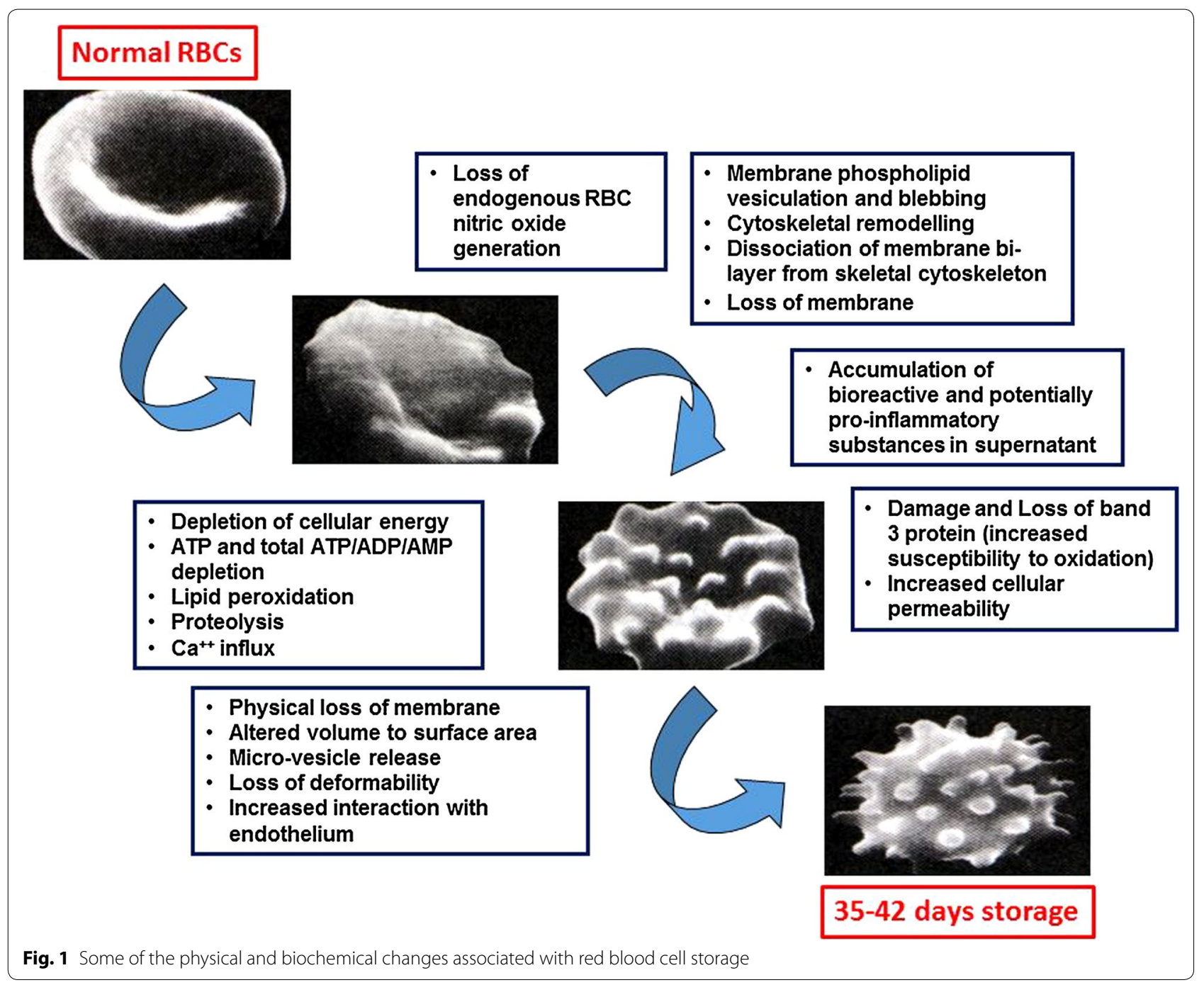

judged at lowest risk of bias (ABLE and TRANSFUSE) the mean pre-transfusion haemoglobin was $76-77 \mathrm{~g} / \mathrm{L}$ indicating evidence-based restrictive practice. ABLE and TRANSFUSE were also double-blind trials, and had very complete follow-up. The trials compared a fresher (6-11 days) with an older (21-24 days) RBC group. The older groups represented 'usual practice' in most healthcare systems, but it is relevant that few patients received RBCs aged more than 30 days when storage changes are most severe. Patients received a mean of four units during ICU stay.

The authors observed no effects from fresher RBCs on mortality, adverse effects or post-transfusion infections in the overall analysis or when restricted to the ABLE and TRANSFUSE trials. Of relevance, statistical heterogeneity for mortality was low indicating consistent findings. The pooled effect size indicated a risk ratio of 1.04 (confidence intervals $0.98-1.10$ ). These point estimates favour older blood and statistically exclude a greater than $2 \%$ benefit from fresher RBCs. The alternative TSA approach concluded that a $10 \%$ relative increase/decrease in relative risk of death from fresher RBCs could be excluded by these data. As mortality was 36\% in ABLE and 24\% in TRANSFUSE, this suggests that effects greater than $3.6 \%$ are highly unlikely. Although statistical certainty for adverse events and post-operative infections was lower, the non-significant point estimates also favoured older RBCs.

We have rarely had such certainty regarding a common critical care intervention. This study provides confidence that requesting fresher $\mathrm{RBCs}$ confers no outcome benefit for our patients, and we should not change our current practice. The point estimate favouring standard age $\mathrm{RBCs}$ is thought-provoking and it is notable that recent observational research, despite its clear limitations, has also shown associations between fresh blood and worse 
outcomes [10]. These trials also do not reassure us that the oldest RBCs are as safe as usual care although a secondary analysis of the INFORM trial found no difference for 'oldest' versus 'freshest' subgroups, which is reassuring. As blood banks usually issue RBCs before these older storage times this is also less clinically relevant. We also cannot be sure about effects during major haemorrhage, where $\mathrm{RBC}$ dose is high and should be cautious assuming these data apply when pre-storage leucodepletion is not standard practice.

In summary, Rygard and colleagues helpfully summarise the substantially increased knowledge regarding clinical effects from RBC storage from the past 3 years, and definitively show that fresh RBCs are neither necessary nor justified during critical illness. There is, however, more research to do for this fundamental healthcare intervention, for example in relation to the importance of donor factors and the way we process RBCs.

\section{Author details}

${ }^{1}$ Department of Anaesthesia, Critical Care and Pain Medicine, University of Edinburgh, Royal Infirmary of Edinburgh, Room S8208, 2nd Floor, 51 Little France Crescent, Edinburgh EH16 4SA, Scotland. ${ }^{2}$ Department of Intensive Care and Laboratory of Experimental Intensive Care and Anesthesiology, Academic Medical Center, Room G3-206, Meibergdreef 9, 1105 AZ Amsterdam, The Netherlands.

\section{Compliance with ethical standards}

\section{Conflicts of interest}

Authors report no conflict of interests.

Received: 12 February 2018 Accepted: 19 February 2018

Published online: 14 March 2018
References

1. Murphy DJ, Needham DM, Netzer G, Zeger SL, Colantuoni E, Ness P, Pronovost PJ, Berenholtz SM (2013) RBC transfusion practices among critically ill patients: has evidence changed practice? Crit Care Med 41:2344-2353

2. Retter A, Wyncoll D, Pearse R, Carson D, McKechnie S, Stanworth S, Allard S, Thomas D, Walsh T (2013) Guidelines on the management of anaemia and red cell transfusion in adult critically ill patients. Br J Haematol 160:445-464

3. Dumont LJ, AuBuchon JP (2008) Evaluation of proposed FDA criteria for the evaluation of radiolabeled red cell recovery trials. Transfusion 48:1053-1060

4. Marik PE, Sibbald WJ (1993) Effect of stored-blood transfusion on oxygen delivery in patients with sepsis. JAMA 269:3024-3029

5. Muszynski JA, Bale J, Nateri J, Nicol K, Wang Y, Wright V, Marsh CB, Gavrilin MA, Sarkar A, Wewers MD, Hall MW (2015) Supernatants from stored red blood cell (RBC) units, but not RBC-derived microvesicles, suppress monocyte function in vitro. Transfusion 55(8):1937-1945

6. Tinmouth A, Fergusson D, Yee IC, Hebert PC (2006) Clinical consequences of red cell storage in the critically ill. Transfusion 46:2014-2027

7. Chasse M, Fergusson DA (2017) Blood donor characteristics on transfusion outcomes-should obesity be assessed in future clinical trials?-reply. JAMA Intern Med 177:599-600

8. Bakkour S, Acker JP, Chafets DM, Inglis HC, Norris PJ, Lee TH, Busch MP (2016) Manufacturing method affects mitochondrial DNA release and extracellular vesicle composition in stored red blood cells. Vox Sang 111:22-32

9. Rygard SL, Jonsson AB, Madsen MB, Perner A, Holst LB, Johansson PI, Wetterslev J (2018) Effects of shorter versus longer storage time of transfused red blood cells in adult ICU patients: a systematic review with metaanalysis and trial sequential analysis. Intensive Care Med 44(2):204-217

10. Heddle NM, Arnold DM, Acker JP, Liu Y, Barty RL, Eikelboom JW, Webert KE, Hsia CC, O'Brien SF, Cook RJ (2016) Red blood cell processing methods and in-hospital mortality: a transfusion registry cohort study. Lancet Haematol 3:e246-e254 